\title{
T3 Subdivision Correlation with Nodal or Distant Metastasis in Colorectal Cancer; Is It Practically Useful?
}

\author{
Nam Kyu Kim \\ Department of Surgery, Yonsei University College of Medicine, Seoul, Korea
}

\section{See Article on Page 160-164}

The pathological staging system has been regarded as a guideline for predicting the prognosis of patients and for deciding on the need for further adjuvant treatment. In this issue, the Yoo et al. [1] analyzed 555 colorectal cancer patients who underwent curative surgery, especially for pT3 cancer subdivided according to the depth of tumor infiltration beyond the muscle proper layer: $<1 \mathrm{~mm}, 1-5 \mathrm{~mm}, 5-15 \mathrm{~mm},>15 \mathrm{~mm}$. These 4 types of pT3 subdivisions were shown to be deeply correlated to the known prognostic histopathologic factors. One thing they did not analyze was the relation between T3 subdivision and the 5-year disease-free survival rate. They only compared pT3 subdivision with nodal and distant metastasis; furthermore, their multivariate analysis revealed that pT3 subtype might be an independent prognostic factor in colorectal cancer. Some studies regarding the relevant risk factors for regional lymph node metastasis in submucosal adenocarcinomas have been performed, and some of those measured not only the submucosal depth of invasion but also tumor budding, lymphatic invasion, vascular invasion, etc. In rectal cancer, the circumferential resection margin (CRM) and tumor deposit also have been reported as important risk factors for predicting patient prognosis, but none of those are recognized as definite prognostic factors in the current American Joint Committee on Cancer (AJCC) staging system. However, several reports have already demonstrated extramural invasion depth to be related with nodal metastasis and distant metastasis in colorectal cancer. Interestingly, pT3 subdivision has been ana-

Correspondence to: Nam Kyu Kim, M.D.

Department of Surgery, Yonsei University College of Medicine, 50 Yonsei-ro, Seodaemun-gu, Seoul 120-749, Korea

Tel: +82-2-2228-2117, Fax: +82-2-313-8289

E-mail:namkyuk@yuhs.ac

(C) 2012 The Korean Society of Coloproctology

This is an open-access article distributed under the terms of the Creative Commons Attribution NonCommercial License (http://creativecommons.org/licenses/by-nc/3.0) which permits unrestricted noncommercial use, distribution, and reproduction in any medium, provided the original work is properly cited. lyzed and has been reported as a prognostic factor in rectal cancer patients not colon cancer patients. Miyoshi et al. [2] analyzed pT3 rectal cancer patients according to a 6-mm cutoff value for the extent of mesorectal invasion, and there were definite differences in the overall 5 -year survival rates between the two groups of patients. Kim et al. [3] reported an interesting study regarding how to select high-risk rectal cancer patients based on preoperative local staging using rectal magnetic resonance imaging (MRI). He compared the extent of mesorectal invasion between whole mounted rectal cancer histopathology and MRI using a 5-mm cutoff value to evaluate its value as a prognostic indicator. The overall accuracy of preoperative MRI was $88 \%$, which means MRI can be used to select patient who show T3 with 5- mm infiltration beyond the muscle layer. Those patients could be candidates for preoperative chemoradiation. Unfortunately, T3 pathological subdivision is not accepted as a prognostic factor in the new AJCC tumornode-metastasis staging system, Furthermore, cutoff points for the penetration depth of cancer invasion beyond the muscle proper layer have not been determined. Currently, most pathologists do not report T3 subdivision, and as far as I can determine, they seem in Korea to be reluctant to measure the distance of tumor infiltration beyond the muscle proper layer. If pT3 subdivision might be a clinically significant prognostic factor, two issues still remain to be solved. One is the lack of a consensus about cutoff points, which should be studied more based on a large patient cohort; the other involves the support and cooperation of pathologists. Based on personal experiences concerning pathological descriptions of the CRM, we have had long discussions and cooperative conferences with pathologists. Currently, CRM status documentation is mandatory in pathological reports submitted in Korea.

I think this study showed us an important message about the heterogeneity of the prognoses for the pT3 group of patients. Conventionally, we have not paid attention to the prognoses of colorectal cancer patients in the same category of pT3 patients. Important feedback to the multidisciplinary team conference can allow patients with high risk of nodal and distant recurrence after curative surgery to be identified. Therefore, the strat- 
egy for treating patients with high risk may be changed based on preoperative modern imaging. Currently, some evidence exists that preoperative chemotherapy or chemoradiotherapy may have oncological benefits compared surgery first $[4,5]$. A couple of well-known high-risk factors are CRM status, tumor deposit, vascular encasement of nodes, etc., and patients with such factors should be candidates for preoperative treatment. Hopefully, pT3 subdivision may be a candidate for use as another high-risk factor, but it seems to have a long way to go.

\section{REFERENCES}

1. Yoo HY, Shin R, Ha HK, Oh HK, Jeong SY, Park KJ, et al. Does T3 Subdivision Correlate with Nodal or Distant Metastasis in Colorectal Cancer? J Korean Soc Coloproctol 2012;28:160-4.

2. Miyoshi M, Ueno H, Hashiguchi Y, Mochizuki H, Talbot IC. Extent of mesorectal tumor invasion as a prognostic factor after $\mathrm{cu}-$ rative surgery for T3 rectal cancer patients. Ann Surg 2006;243:
492-8.

3. Kim YW, Cha SW, Pyo J, Kim NK, Min BS, Kim MJ, et al. Factors related to preoperative assessment of the circumferential resection margin and the extent of mesorectal invasion by magnetic resonance imaging in rectal cancer: a prospective comparison study. World J Surg 2009;33:1952-60.

4. Pedersen BG, Moran B, Brown G, Blomqvist L, Fenger-Grøn M, Laurberg S. Reproducibility of depth of extramural tumor spread and distance to circumferential resection margin at rectal MRI: enhancement of clinical guidelines for neoadjuvant therapy. AJR Am J Roentgenol 2011;197:1360-6.

5. Dewdney A, Cunningham D, Tabernero J, Capdevila J, Glimelius B, Cervantes A, et al. Multicenter randomized phase II clinical trial comparing neoadjuvant oxaliplatin, capecitabine, and preoperative radiotherapy with or without cetuximab followed by total mesorectal excision in patients with high-risk rectal cancer (EXPERT-C). J Clin Oncol 2012;30:1620-7. 